\title{
High Throughput Radix-4 SISO Decoding Architecture with Reduced Memory Requirement
}

\author{
Wooseok Byun, Hyeji Kim, and Ji-Hoon Kim
}

\begin{abstract}
As the high-throughput requirement in the next generation communication system increases, it becomes essential to implement high-throughput SISO (Soft-Input Soft-Output) decoder with minimal hardware resources. In this paper, we present the comparison results between cascaded radix-4 ACS (Add-Compare-Select) and LUT (Look-Up Table)based radix-4 ACS in terms of delay, area, and power consumption. The hardware overhead incurred from the retiming technique used for high speed radix-4 ACS operation is also analyzed. According to the various analysis results, high-throughput radix-4 SISO decoding architecture based on simple path metric recovery circuit is proposed to minimize the hardware resources. The proposed architecture is implemented in $65 \mathrm{~nm}$ CMOS process and memory requirement and power consumption can be reduced up to $78 \%$ and $32 \%$, respectively, while achieving high-throughput requirement.
\end{abstract}

Index Terms-Add-compare-select, high-throughput, retiming technique, SISO decoder, signal processing

\section{INTRODUCTION}

ACS units which compose the SISO decoder play the key role in many FEC (Forward Error Correction) decoders such as Viterbi decoder and Turbo decoder. As the throughput requirement for building infrastructure of

Manuscript received Feb. 12, 2014; accepted Jul. 14, 2014 A part of this work was presented in International SoC Design Conference (ISOCC), Busan in Korea, November 2013.

Chungnam National University.

E-mail : jihoonkim@cnu.ac.kr the communication increases, it becomes more important to implement the high speed ACS unit which usually limits the maximum operating frequency.

The ACS unit consists of three basic computations such as addition, comparison, and selection. The purpose of ACS operation is updating the path metrics, recursively, using the addition of path metric and branch metric, and the selection of maximum or minimum value among the addition results. In this paper, the maximum selection is exploited but the proposed architectures can be applicable to the minimum selection algorithm. This whole operation for a conventional cascaded radix-4 ACS unit can be represented by the following equation;

$$
\begin{gathered}
P_{N E W}=\max \left\{\max \left(P_{A}, P_{B}\right), \max \left(P_{C}, P_{D}\right)\right\} \\
\text { where } P_{X}=P_{k}+\gamma_{k}
\end{gathered}
$$

where $P_{N E W}$ is output value of radix-4 ACS unit, which denotes the original path metric to be updated. $P_{X}$ is the pre-compare metric where the $X$ belongs to $\{A, B$, $C, D\}$. The $P_{k}$ and the $\gamma_{k}$ are the path metric and the branch metric, respectively, where the $k$ belongs to $\{0$, $1,2,3\}$. The elements of $X$ and $k$ are matched sequentially. These pre-compare metrics are the sum of path metric and branch metric. Eq. (1) indicates the maximum value can be obtained from two local maximum values.

This recursive operation feature leads to the long critical path delay of radix-4 ACS unit, and becomes the bottleneck and the throughput limitation of SISO decoders. Outputs of ACS unit could not be obtained by applying the pipeline architecture to the ACS unit since ACS units perform the recursive operation caused by 
Markov chain.

To increase the throughput of SISO decoders, there are several methods such as the increase of parallelism of decoder [1-10], the improvement of operating frequency [11], and the high radix architecture [12-14]. This paper is focused on high operating frequency of radix-4 ACS unit for the high-throughput of radix-4 SISO decoders.

This paper is organized as follows. In Section II, the retimed cascaded radix -4 ACS and retimed LUT-based radix-4 ACS with several signal processing techniques were comparatively explained. In Section III, to reduce the memory requirement of both retimed ACS units, memory requirement of radix-4 SISO decoder was introduced and proposed memory reduction technique for path metrics which are also called forward/backward metrics was presented. The comparison of implementation results between the cascaded radix-4 ACS units and the LUT-based radix-4 ACS units in Samsung $65 \mathrm{~nm}$ CMOS process were presented in Section IV with reduced memory requirement and power consumption.

\section{High SPEED ACS UNIT ARCHITECTURE}

To reduce the critical path delay of high-radix ACS unit, several signal processing techniques can be applied such as common operator migration and retiming [15]. Application of these signal processing techniques to widely used previous radix-4 ACS architectures such as conventional cascaded radix-4 ACS and LUT-based radix-4 ACS is introduced as follows [17]. In this paper, the word length of all kind of metrics is set to 12-bit.

\section{Retiming of Cascaded Radix-4 ACS Unit}

As indicated in Fig. 1(each component name is described in Fig. 2(b)), Type-C1, the conventional radix-4 ACS unit which consists of two cascaded 2-input ACS units denotes the most straightforward hardware architecture of Eq. (1). More precisely, Type-C1 has following equation.

$$
\begin{gathered}
P_{\text {NEW.C1 }}=\max \left\{\max \left(P_{0}+\gamma_{0}, P_{1}+\gamma_{1}\right),\right. \\
\left.\max \left(P_{2}+\gamma_{2}, P_{3}+\gamma_{3}\right)\right\}-P_{N O R M}
\end{gathered}
$$

Each hardware component has computational latency

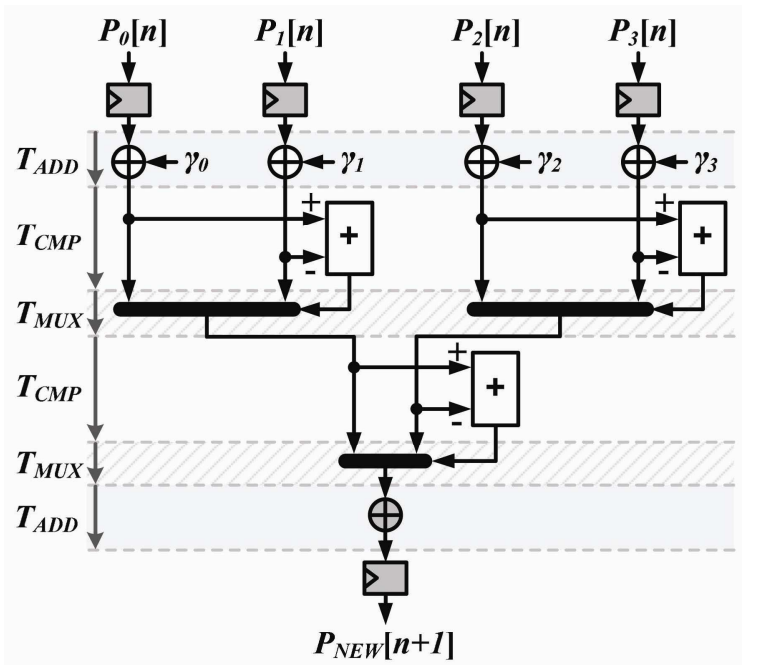

Fig. 1. Architecture of Type-Cl: the conventional cascaded radix-4 ACS unit.

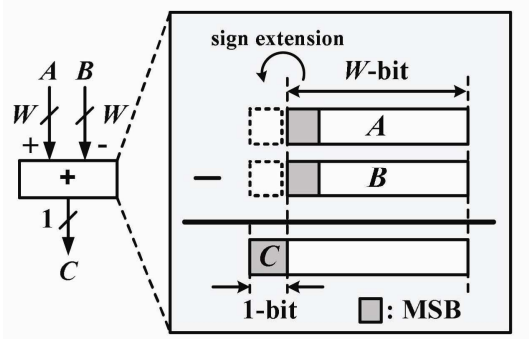

(a)

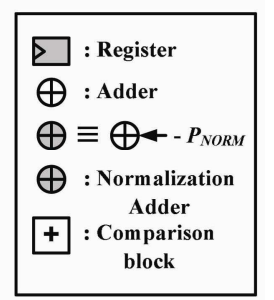

(b)
Fig. 2. Illustration of (a) comparing procedure inside the comparison block, (b) legend describing the name of hardware components. $P_{N O R M}$ denotes the normalization metric.

represented by $T$ with subscript. The critical path delay of the Type-C1, $T_{C R I T . C 1}$, is represented as

$$
T_{C R I T . C 1}=2 T_{A D D}+2 T_{C M P}+2 T_{M U X}
$$

where $T_{A D D}, T_{C M P}$, and $T_{M U X}$ indicates the computational latency of adder, comparison block, and multiplexor (MUX), respectively. In Fig. 2(a), the comparison block has $T_{C M P}$ latency. The most significant bit (MSB) of output value $C$ represents the sign of the result and indicates whether or not the second input is bigger than the first input.

In Type-C1, there is no concurrent processing through the whole computation path. All operations are performed sequentially. This operation scheme characterizes the architecture that has long critical path delay. For the improved decoding throughput, several 


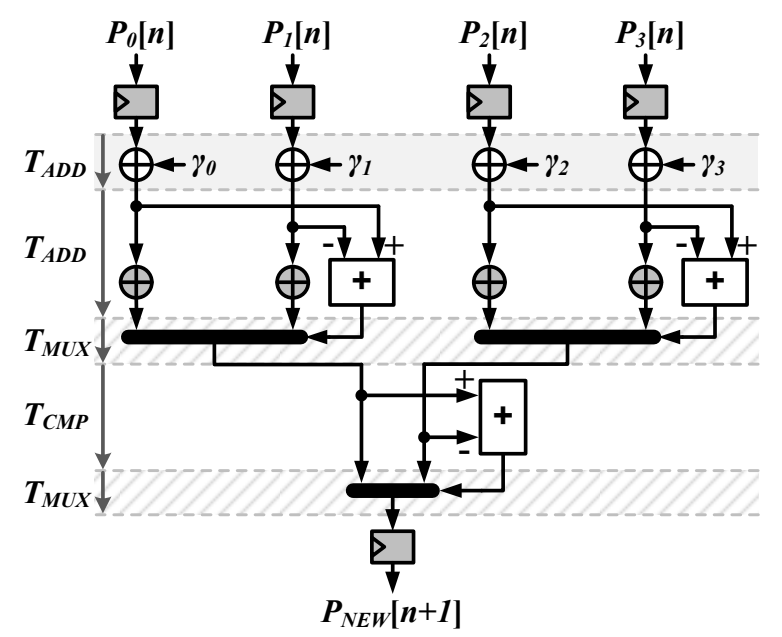

Fig. 3. Architecture of Type-C1-A: modified conventional cascaded radix-4 ACS unit with common operator migration.

hardware components should perform their operations, concurrently.

To reduce the critical path delay, common operation which is equally applied to all of the computation paths can be moved to other place and performed with other operations, concurrently. In Fig. 1, all computation paths of Type-Cl have normalization adder which can be moved between the branch metric adder and the first stage 2-to-1 MUX. The result of moving the normalization adder becomes Type-C1-A as indicated in Fig. 3. The result of common operator migration, Type$C 1-A$, has following equation.

$$
\begin{aligned}
& P_{\text {NEW.C1A }} \\
& =\max \left\{\max \left(P_{0}+\gamma_{0}-P_{N O R M}, P_{1}+\gamma_{1}-P_{N O R M}\right),\right. \\
& \left.\quad \max \left(P_{2}+\gamma_{2}-P_{N O R M}, P_{3}+\gamma_{3}-P_{N O R M}\right)\right\}
\end{aligned}
$$

The normalization adder is concurrently operated with comparison block. Since the computational delay of adder is bigger than that of comparison block in $65 \mathrm{~nm}$ CMOS process as indicated in the bottom of Fig. 8, even though the comparison block completes its operation, the first stage MUX of Type-C1-A should wait for normalization adder to select the accurate value. For this reason, $T_{A D D}$ should be considered rather than $T_{C M P}$ to define the critical path delay in Type-C1-A. The computational latency $T_{C M P}$ is reduced from Type-C1 and the critical path delay of Type-C1-A represented as below.

$$
\begin{aligned}
T_{C R I T . C 1 A} & =T_{C R I T . C 1}-T_{C M P} \\
& =2 T_{A D D}+T_{C M P}+2 T_{M U X}
\end{aligned}
$$

This signal processing technique, so called common operator migration, brings reduced critical path delay in comparison with Eq. (3).

Retiming is the transformation technique which can be used to change the locations of registers in a circuit without affecting the input/output characteristics of the circuit [15]. In Fig. 3, the top/bottom registers cut off the recursive operation of radix-4 ACS unit. If this recursive operation was represented in the form of loop scheme, there will be a one register through the entire computation loop because the $P_{N E W}[n+1]$ in Type-C1$A$ denotes the output value of certain register located in the top of Fig. 3 in time instance $n+1$. For applying common operator migration again to Type-Cl-A, it is necessary to change locations of several registers for parallel process of several hardware components. In Fig. 3 , registers of Type-C1-A can be moved between branch metric adder and comparison block of first stage. According to the migration of registers, that is retiming, the comparison in the second stage and branch metric addition can be performed directly without waiting for the clock edge as shown in Fig. 4(a). Finally, common operator migration of branch metric addition to the location before the second stage MUX of Type-Cl-B brings reduced critical path delay amount of $T_{C M P}$ and this retimed architecture is described in Fig. 4(b) as Type-C2. In the retimed radix-4 ACS unit, the metrics to be stored in forward memory are not the original path metrics. These four metrics are recursive path metrics and denoted as $R_{k}$ where $k$ belongs to $k=\{0,1,2,3\}$. The nodes before the branch metric calculation in the retimed radix-4 ACS unit is called $R_{\alpha}$ and $R_{\beta}$, respectively. The equation of Type-C2 operation and its critical path delay is represented as below.

$$
\begin{gathered}
R_{\alpha}=\max \left(R_{0}[n]-P_{\text {NORM }}, R_{1}[n]-P_{\text {NORM }}\right) \\
R_{\beta}=\max \left(R_{2}[n]-P_{\text {NORM }}, R_{3}[n]-P_{\text {NORM }}\right) \\
R_{k}[n+1]=\max \left(R_{\alpha}+\gamma_{k}, R_{\beta}+\gamma_{k}\right) \\
T_{\text {CRIT.C2 }}=T_{C R I T . C 1 A}-T_{C M P} \\
=2 T_{A D D}+2 T_{M U X}
\end{gathered}
$$

It is clear that the critical path delay of Type-C2, Eq. 


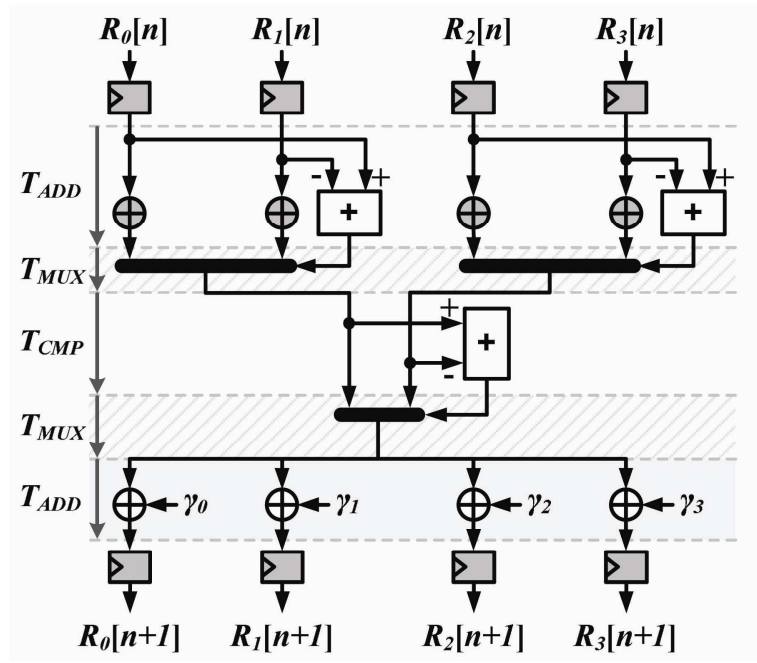

(a)

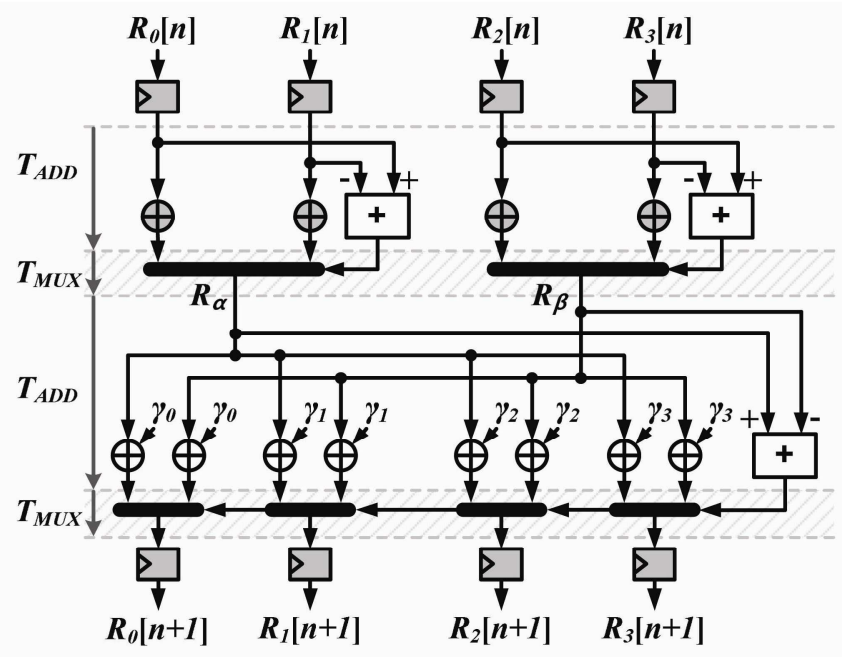

(b)

Fig. 4. Modified architectures of Type-C1-A with applying the retiming technique (a) Type-C1-B: the register moved architecture, (b) Type-C2: the retimed architecture which is obtained through the common operator migration on the Type-C1-B.

(7), is smaller than the both of Eqs. (3) and (5).

Even though Type-C1 is transformed into Type-C2 with reducing the computational latency amount of $2 T_{C M P}$ through several signal processing techniques, Type-C2 still maintains its cascaded hardware architecture. It cannot complete a one iteration of computation until both the two stage are completely operated sequentially.

\section{Retiming of LUT-Based Radix-4 ACS Unit}

Fig. 5 shows the basic form of LUT-based radix-4 ACS unit, Type-L1, which contains the LUT combinational logic [17]. It is not cascaded form of three 2-input ACS units but single form of 4-input ACS unit which computes the four path metrics and branch metrics at once. This section explains briefly the algorithm of LUT-based radix4 ACS operation and presents the results of applying signal processing techniques to the Type-L1.

After the addition between the path metrics $\left(P_{0}[n]\right.$ to $\left.P_{3}[n]\right)$ and the branch metrics $\left(\gamma_{0}\right.$ to $\left.\gamma_{3}\right)$, the four addition results which are called pre-compare metrics ( $P_{A}$ to $P_{D}$ ) should be compared for finding the maximum value. As indicated in Fig. 5, the pre-compare metrics are the four results of addition between the path metrics and the branch metrics at time instance $n$. To find the maximum value among four pre-compare metrics, the comparison blocks should compare each magnitude of

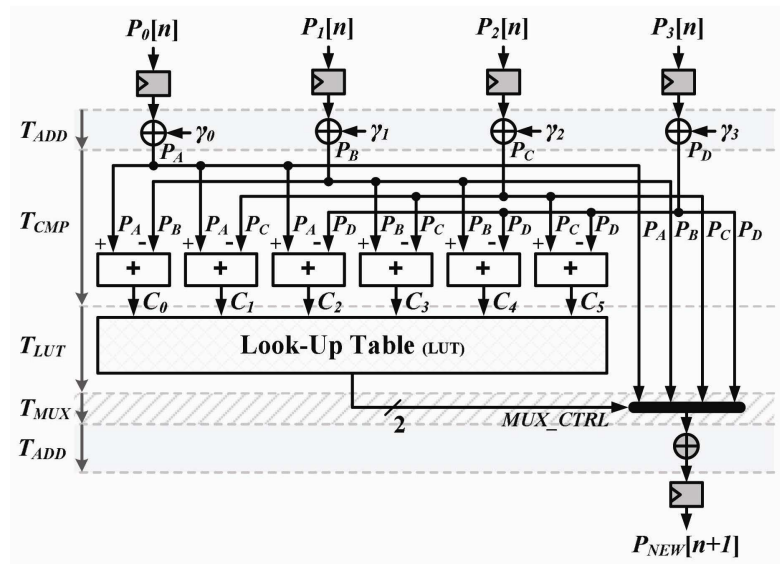

Fig. 5. Architecture of Type-L1: the basic form of LUT-based radix-4 ACS unit.

them and find out the relationship as indicated in [17]. This Type-L1 has following equation.

$$
\begin{aligned}
P_{N E W . L 1}= & \max \left(P_{0}+\gamma_{0}, P_{1}+\gamma_{1},\right. \\
& \left.P_{2}+\gamma_{2}, P_{3}+\gamma_{3}\right)-P_{N O R M}
\end{aligned}
$$

To obtain all the magnitude relationships of different four pre-compare metrics, it is required the number of combinations of four different pre-compare metrics, taken two at a time, without repetitions as represented by the following equation;

$$
\left(\begin{array}{l}
n \\
k
\end{array}\right)=\frac{n !}{k !(n-k) !}
$$


Table 1. Four groups of magnitude-ordered combinations

\begin{tabular}{|c|c|c|c|c|c|c|c|c|c|c|c|c|c|c|c|}
\hline & \multicolumn{9}{|c|}{ G1 } & \multicolumn{4}{c|}{ G2 } & G3 & G4 \\
\hline Composition Name & S1 & S2 & S3 & S4 & S5 & S6 & S7 & S8 & S9 & S10 & S11 & S12 & S13 & S14 & S15 \\
\hline$P_{A}$ & $\bullet$ & & & $\bullet$ & & $\bullet$ & $\bullet$ & $\vee$ & $\vee$ & $\bullet$ & & $\bullet$ & $\bullet$ & $\bullet$ & \\
\hline$P_{B}$ & $\bullet$ & $\bullet$ & & & $\bullet$ & & $\bullet$ & $\bullet$ & $\bullet$ & $\bullet$ & $\bullet$ & & $\bullet$ & $\bullet$ & \\
\hline$P_{C}$ & & $\bullet$ & $\bullet$ & & & $\bullet$ & $\vee$ & $\bullet$ & $\vee$ & $\bullet$ & $\bullet$ & $\bullet$ & & $\bullet$ & \\
\hline$P_{D}$ & & & $\bullet$ & $\bullet$ & $\bullet$ & & $\vee$ & $\vee$ & $\bullet$ & & $\bullet$ & $\bullet$ & $\bullet$ & $\bullet$ & \\
\hline
\end{tabular}

*G1: 1st group, G2: 2nd group, ,.., G4: 4th group

*If several pre-compare metrics have the same mark in each composition, these are repetitions.

*All blank cells indicate non repetitions in each composition.

Table 2. Compositions of six MSBs for all combinations of pre-compare metrics

\begin{tabular}{|c|c|c|c|c|c|c|c|}
\hline & Order of $P_{\{\mathrm{X}\}}$ & $C_{0}$ & $C_{1}$ & $C_{2}$ & $C_{3}$ & $C_{4}$ & $C_{5}$ \\
\hline \multirow{4}{*}{$\mathrm{S} 1$} & (A B) C D & 0 & 0 & 0 & 0 & 0 & 0 \\
\cline { 2 - 8 } & (A B) D C & 0 & 0 & 0 & 0 & 0 & 1 \\
\cline { 2 - 8 } & $\mathrm{C} \mathrm{(A} \mathrm{B)} \mathrm{D}$ & 0 & 1 & 0 & 1 & 0 & 0 \\
\cline { 2 - 8 } & $\mathrm{C} \mathrm{D} \mathrm{(A} \mathrm{B)}$ & 0 & 1 & 1 & 1 & 1 & 0 \\
\cline { 2 - 8 } & $\mathrm{D}(\mathbf{A ~ B ) ~ C}$ & 0 & 0 & 1 & 0 & 1 & 1 \\
\cline { 2 - 8 } & $\mathrm{D} \mathrm{C} \mathrm{(A} \mathrm{B)}$ & 0 & 1 & 1 & 1 & 1 & 1 \\
\hline
\end{tabular}

\begin{tabular}{|l|c|c|c|c|c|c|c|}
\hline & Order of $P_{\{\mathrm{X}\}}$ & $C_{0}$ & $C_{1}$ & $C_{2}$ & $C_{3}$ & $C_{4}$ & $C_{5}$ \\
\hline \multirow{2}{*}{ S7 } & (A B) (C D) & 0 & 0 & 0 & 0 & 0 & 0 \\
\cline { 2 - 8 } & (C D) (A B) & 0 & 1 & 1 & 1 & 1 & 0 \\
\hline \multirow{2}{*}{ S10 } & (A B C) D & 0 & 0 & 0 & 0 & 0 & 0 \\
\cline { 2 - 8 } & D (A B C) & 0 & 0 & 1 & 0 & 1 & 1 \\
\hline S14 & (A B C D) & 0 & 0 & 0 & 0 & 0 & 0 \\
\hline
\end{tabular}

*The first column represents the composition name of Table 1.

where $n$ is the number of different things and $k$ is the number of values taken at a time. For the result of Eq. (9) where $n$ is 4 and $k$ is 2 , all magnitude relationships are come out through the six combinations. These combinations perform the comparison as indicated in Fig. 2(a) and its six results, $C_{0}$ to $C_{5}$, represent whether or not the second input is bigger than the first input.

To perform the six comparison operations, the six pairs of pre-compare metrics and the comparison results are defined as follows;

$$
\begin{aligned}
& C_{0}=\operatorname{MSB}\left(P_{A}-P_{B}\right) \\
& C_{1}=\operatorname{MSB}\left(P_{A}-P_{C}\right) \\
& C_{2}=\operatorname{MSB}\left(P_{A}-P_{D}\right) \\
& C_{3}=\operatorname{MSB}\left(P_{B}-P_{C}\right) \\
& C_{4}=\operatorname{MSB}\left(P_{B}-P_{D}\right) \\
& C_{5}=\operatorname{MSB}\left(P_{C}-P_{D}\right)
\end{aligned}
$$

where all the $C_{k}(0 \leq k \leq 5)$ have 1-bit width and $C=M S B(A-B)$ operation is described in Fig. 2(a). The set of $\left\{C_{0}, C_{1}, C_{2}, C_{3}, C_{4}, C_{5}\right\}$ can have the number of $2^{6}$ cases. The MUX_CTRL signal in LUT-based radix-4 ACS unit denotes the MUX control signal to select the maximum value among four inputs of MUX. The maximum value among the four pre-compare metrics is determined by the composition of $\left\{C_{0}, C_{1}, C_{2}, C_{3}, C_{4}\right.$, $\left.C_{5}\right\}$ and pointed by $M U X_{-} C T R L$ encoded into 00, 01, 10,
11 for $P_{A}, P_{B}, P_{C}, P_{D}$, respectively. This whole information is converted into the truth table.

The process of finding the maximum value using six MSBs is described following. To find the entire combinations of four pre-compare metrics in magnitude order, repetitions should be considered. Table 1 denotes entire available compositions of pre-compare metrics with considering the cases when some pre-compare metrics have the same value. As indicated in Table 1, there are three groups of magnitude-ordered combinations with repetitions. The elements of first group have a pair of two same metrics or two pair of two same metrics. There are three same metrics and one different metric in second group, and four same metrics in third group. In addition, there is the 4th group which has no repetitions and the number of its elements is the number of permutations of four different pre-compare metrics taken all at a time. The number of these permutations is represented by factorial 4 !. Table 2 represents only four examples among the all of these combinations with the composition of six MSBs. All of these combinations of three groups and 24 permutations are valid. Except these valid combinations of precompare metrics and its matched composition of six MSBs, there are a lot of invalid cases. These invalid cases will never occur logically. This truth table only determines the maximum value of four pre-compare metrics, it cannot find out the second biggest value. 


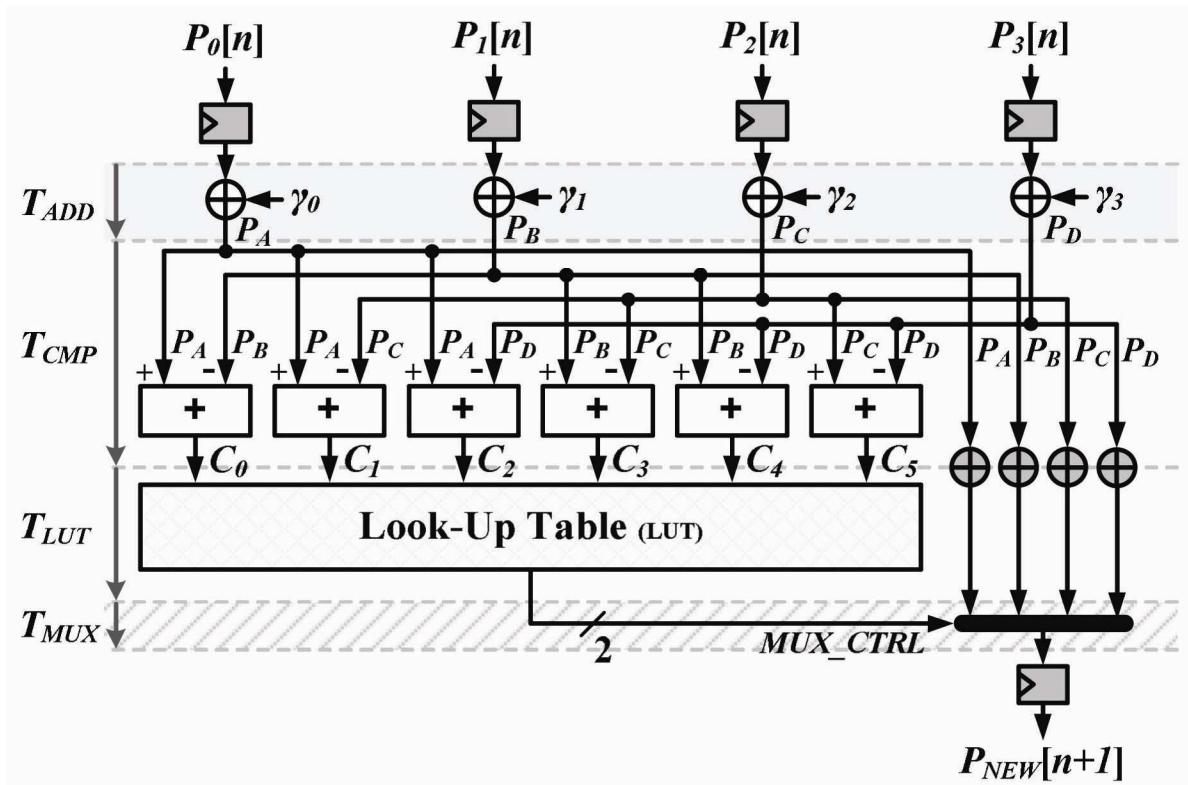

Fig. 6. Architecture of Type-L1-A: modified original LUT-based radix-4 ACS unit with common operator migration.

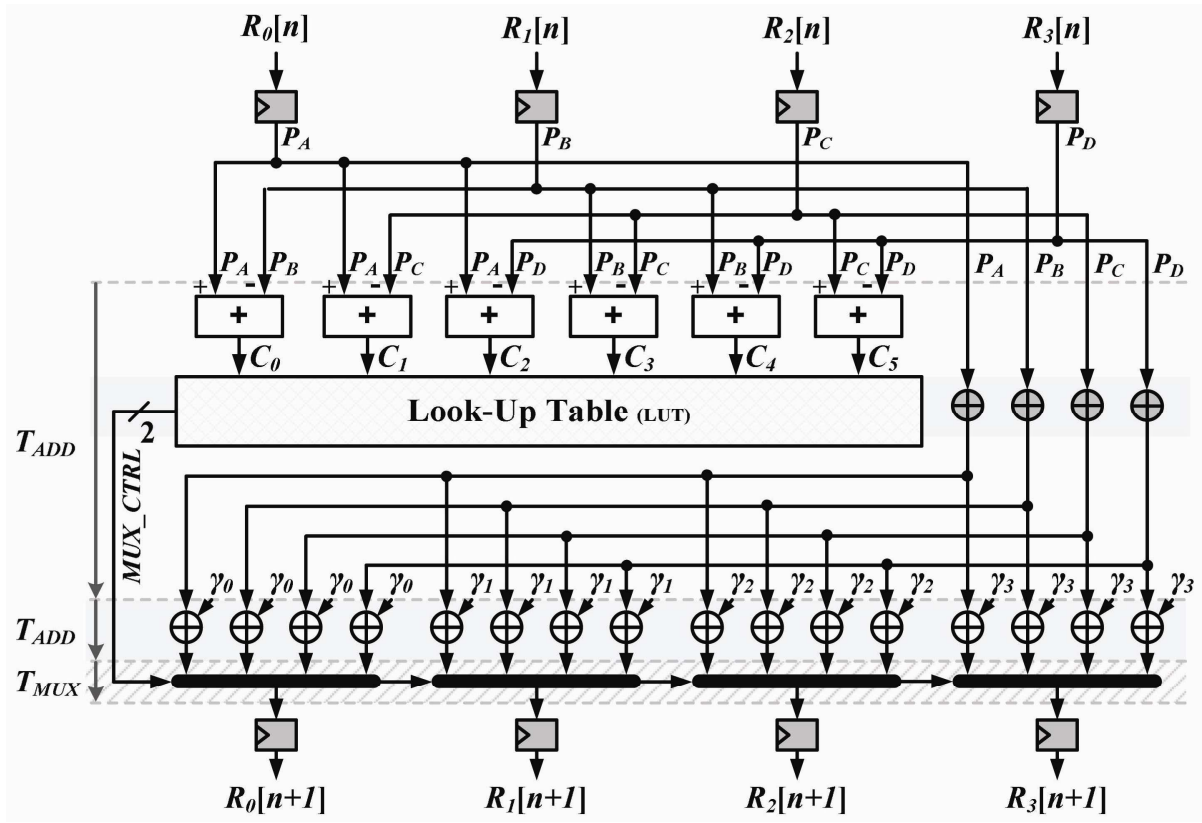

Fig. 7. Architecture of Type-L2: modified Type-L1-A by applying the retiming technique.

To reduce the critical path delay, the signal processing techniques explained in the previous section can be also applied to Type-L1.

Fig. 6 and Eq. (11) represent the Type-L1-A which is the result of applying common operator migration to Type-L1. The normalization adder of Type-L1 which is lastly performed for updating the path metric is moved between the branch metric adder and the 4-to-1 MUX to reduce the computational latency $T_{A D D}$ as following equation;

$$
\begin{aligned}
& P_{N E W . L 1 A} \\
& \begin{aligned}
\max \left(P_{0}+\gamma_{0}-P_{N O R M}, P_{1}+\gamma_{1}-P_{N O R M}\right. \\
\left.\quad P_{2}+\gamma_{2}-P_{N O R M}, P_{3}+\gamma_{3}-P_{N O R M}\right)
\end{aligned} \\
& T_{C R I T . L 1}=2 T_{A D D}+T_{C M P}+T_{L U T}+T_{M U X} \\
& T_{C R I T . L 1 A}=T_{C R I T . L 1}-T_{A D D} \\
& =T_{A D D}+T_{C M P}+T_{L U T}+T_{M U X}
\end{aligned}
$$


where $T_{L U T}$ indicates the computational latency of LUT combinational logic. Between the operations of branch metric adder and 4-to-1 MUX, comparison block and LUT combinational logic were performed with involving the normalization addition $T_{A D D}<T_{C M P}+T_{L U T}$.

After the Type-L1-A is obtained through common operator migration, the critical path delay also can be reduced using retiming technique. As indicated in Fig. 7, registers in Type- $L 1-A$ are moved to the node which denotes the pre-compare metrics. Finally, the branch metric adders which are the common operation of Type$L 1-A$ for each branch metrics are moved to the in front of 4-to-1 MUX and these were performed with comparison block and LUT combinational logic, concurrently. The result of retiming to Type-L1-A, Type-L2, is indicated in Fig. 7, and it has following equation where the $k$ belongs to $\{0,1,2,3\}$.

$$
\begin{aligned}
& R_{k}[n+1] \\
& =\max \left(R_{0}[n]-P_{\text {NORM }}+\gamma_{k}, R_{1}[n]-P_{\text {NORM }}+\gamma_{k},\right. \\
& \left.\quad R_{2}[n]-P_{\text {NORM }}+\gamma_{k}, R_{3}[n]-P_{\text {NORM }}+\gamma_{k}\right)
\end{aligned}
$$

In Type-L2, there are two data paths for completing the whole computations. One of them is [comparison block $\rightarrow$ LUT combinational logic $\rightarrow M U X]$ path and the other is [normalization adder $\rightarrow$ branch metric adder $\rightarrow M U X$ ] path. To determine the computational latency between the node of pre-compare metrics and MUX, the longest delay path should be determined first. From a commonsense point of view, since LUT combinational logic has six 1-bit inputs and a 2-bit output in contrast with the branch metric adder or normalization adder which has two 12-bit inputs and a 12-bit output, it is clearly determined what has smaller computational latency. The LUT combinational logic has smaller delay compared to adders and it brings the reduced critical path delay as follows;

$$
\begin{aligned}
T_{C R I T . L 2} & =T_{C R I T . L 1 A}-T_{A D D}+2 T_{A D D}-\left(T_{C M P}+T_{L U T}\right) \\
& =2 T_{A D D}+T_{M U X}
\end{aligned}
$$

where $2 T_{A D D}-\left(T_{C M P}+T_{L U T}\right)$ is the additional delay caused by migrating the branch metric adder.

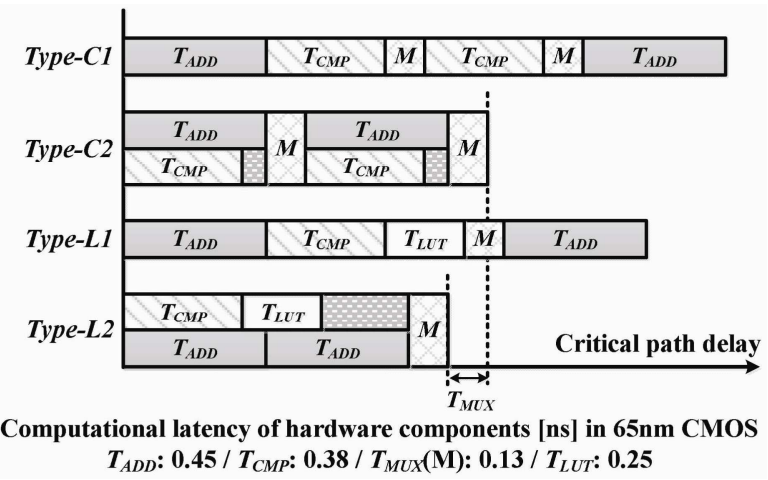

Fig. 8. Prediction of critical path delay for each type represented as Eqs. (3), (7), (12) and (14) based on Samsung 65 nm CMOS process.

\section{Comparative Analysis}

For the comparative analysis of four radix-4 ACS units (Type-C1, Type-C2, Type-L1, and Type-L2), Fig. 8 illustrates the critical path delay with computational latency of hardware components. In Fig. 8, four bar graphs describe computation flow of ACS operation and denote equations of critical path delay indicated in Eqs. (3), (7), (12) and (14). Type-L2 has the shortest critical path delay which is obtained by comparing the magnitude of six pre-compare metrics all together. As indicated in Fig. 4(b) and Fig. 8, Type-C2, the retimed form of cascaded radix-4 ACS unit performs the operations of adder and MUX twice. It is an obstacle in reducing the critical path delay.

\section{Proposed RAdiX-4 SISO Decoding ARCHITECTURE}

By applying several signal processing techniques, the both retimed results, Type-C2 and Type-L2, can be the most appropriate ACS unit to achieve high-throughput radix-4 SISO decoder. However, even though the SISO decoding architecture uses sliding window technique, the SISO decoder which consists of Type-C2 or Type-L2 requires huge memory resources to store path metrics and branch metrics. To reduce the branch metric memory requirement, previous work [16] can be exploited. This paper proposes the memory reduction technique for forward memory in MAP(maximum a posteriori)-based radix-4 SISO algorithm for turbo decoder. 


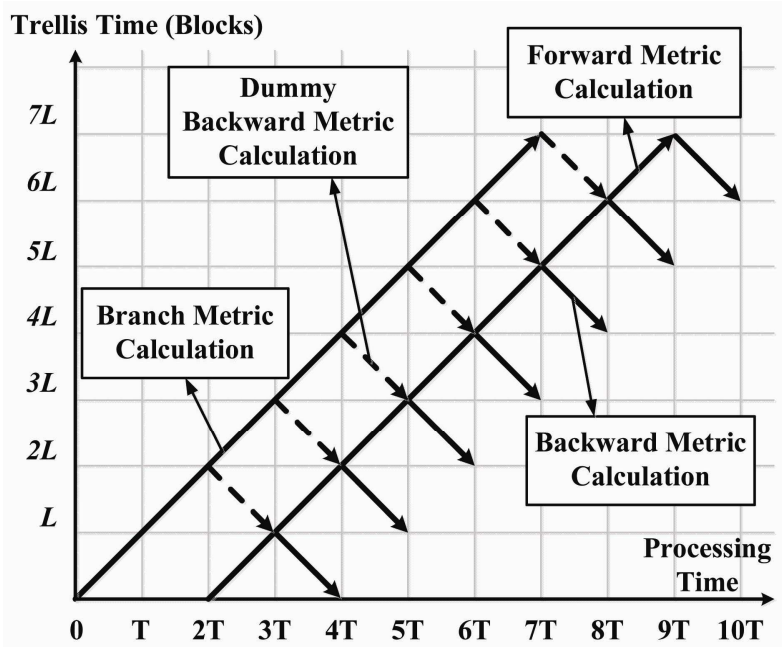

Fig. 9. Sliding window diagram with dummy calculation for indicating the memory requirement of upward and downward directions.

\section{Memory Requirement of Radix-4 SISO Decoder}

As indicated in Fig. 9, the sliding window technique, where a large frame is split into a number of small windows and the decoding algorithm is applied to each window independently, is used for reducing the memory size required to store path metrics [16]. The forward metrics are calculated prior to backward metrics and these will be stored on forward memory. To obtain reliability on error rate, the window size $L$ is set to 64 . Since the initial values at the border of each window should have reliability, the dummy calculation is performed for the backward metrics. To perform the path metric related calculation, there are two memory banks and the forward memory requirement for a radix-4 SISO decoder can be found as Table 3. Each retimed ACS unit, Type-C2 and Type-L2, has four outputs which will be stored in forward memory. Since the radix-4 SISO decoder requires eight radix-4 ACS units for upward direction and eight radix-4 ACS units for downward direction, one radix-4 SISO decoder has sixteen radix-4 ACS units for the path metric calculation. In brief, the number of path metrics which will be stored increased four times, from 8 to 32 , in consequence of retiming.

\section{Proposed Memory Reduction Technique}

In the retimed ACS units, the metrics to be kept in the registers during the recursion are different from original path metrics as indicated in Figs. 4(b) and 7. Therefore, as explained above, the number of metrics to be stored in the forward memory during the recursion increases from 8 to 32 . To reduce the forward memory requirement, we propose the simple recovery circuit to retrieve the original path metric as shown in Figs. 10 and 11. We obtain the original path metric from the node before the branch metric addition.

As indicated in Figs. 10 and 11, $P_{N E W}[n+1]$ is the recovered metric which is the original path metric to be updated and to be stored in forward memory. The recursive path metrics, $R_{0}[n+1]$ to $R_{3}[n+1]$, are not stored in forward memory and temporarily kept during the recursion. Each operational equation of recovery

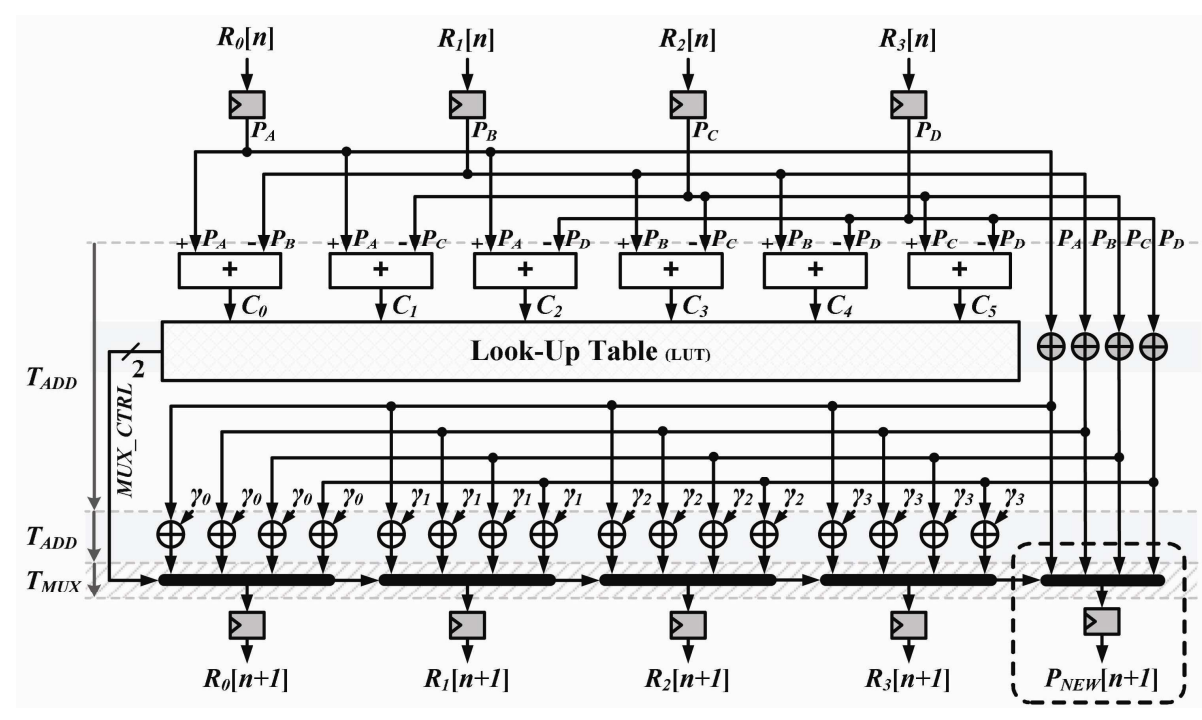

Fig. 10. Architecture of Type-L3: modified Type-L2 with adding the recovery circuit which is shown in dotted line. 
Table 3. Forward memory requirement for a radix-4 SISO decoder and comparison results between the cascaded ACS units and the LUT-based ACS units for path metric calculation

\begin{tabular}{|c|c|c|c|c|c|}
\hline & \multicolumn{2}{|c|}{ Cascaded ACS } & \multicolumn{2}{|c|}{ LUT-based ACS } \\
\hline & & $\begin{array}{c}\text { Type-C2 } \\
\left(32^{\dagger}\right)\end{array}$ & $\begin{array}{c}\text { Type-C3 } \\
\left(7^{\dagger}\right)\end{array}$ & $\begin{array}{c}\text { Type-L2 } \\
\left(32^{\dagger}\right)\end{array}$ & $\begin{array}{c}\text { Type-L3 } \\
\left(7^{\dagger}\right)\end{array}$ \\
\hline \multirow{2}{*}{\multicolumn{2}{|c|}{$\begin{array}{c}\text { Forward } \\
\text { Memory } \\
\text { Requirement }\end{array}$}} & $\begin{array}{c}2 \text { banks, } \\
64 *(12 * 32) \\
\text { bits/bank }\end{array}$ & $\begin{array}{c}2 \text { banks, } \\
64 *(12 * 7) \\
\text { bits/bank }\end{array}$ & $\begin{array}{c}2 \text { banks, } \\
64 *(12 * 32) \\
\text { bits/bank }\end{array}$ & $\begin{array}{c}2 \text { banks, } \\
64 *(12 * 7) \\
\text { bits/bank }\end{array}$ \\
\hline & & $\begin{array}{c}49152 \text { bits } \\
(100 \%)\end{array}$ & $\begin{array}{c}10752 \text { bits } \\
(22 \%)\end{array}$ & $\begin{array}{c}49152 \text { bits } \\
(100 \%)\end{array}$ & $\begin{array}{c}10752 \text { bits } \\
(22 \%)\end{array}$ \\
\hline $\begin{array}{c}\text { Gate } \\
\text { Count } \\
{[\mathrm{K}]}\end{array}$ & $\begin{array}{l}\text { ACS } \\
\text { Units }\end{array}$ & $\begin{array}{c}44.40 \\
(100 \%)\end{array}$ & $\begin{array}{c}46.92 \\
(106 \%)\end{array}$ & $\begin{array}{c}57.78 \\
(100 \%)\end{array}$ & $\begin{array}{c}60.12 \\
(104 \%)\end{array}$ \\
\hline \multirow{3}{*}{$\begin{array}{l}\text { Area } \\
{\left[\mu \mathrm{m}^{2}\right]}\end{array}$} & $\begin{array}{l}\text { ACS } \\
\text { Units }\end{array}$ & $\begin{array}{c}56827 \\
(100 \%)\end{array}$ & $\begin{array}{c}60058 \\
(106 \%)\end{array}$ & $\begin{array}{c}73953 \\
(100 \%)\end{array}$ & $\begin{array}{c}76954 \\
(104 \%)\end{array}$ \\
\hline & Mem. & $\begin{array}{l}125687 \\
(100 \%)\end{array}$ & $\begin{array}{l}37281 \\
(30 \%)\end{array}$ & $\begin{array}{l}125687 \\
(100 \%)\end{array}$ & $\begin{array}{l}37281 \\
(30 \%)\end{array}$ \\
\hline & Total & $\begin{array}{l}182514 \\
(100 \%)\end{array}$ & $\begin{array}{l}97339 \\
(53 \%)\end{array}$ & $\begin{array}{l}199641 \\
(100 \%)\end{array}$ & $\begin{array}{c}114235 \\
(57 \%)\end{array}$ \\
\hline \multirow{3}{*}{$\begin{array}{l}\text { Power } \\
{[\mathrm{mW}]}\end{array}$} & $\begin{array}{l}\text { ACS } \\
\text { Units }\end{array}$ & $\begin{array}{c}36.96 \\
(100 \%)\end{array}$ & $\begin{array}{c}40.51 \\
(110 \%)\end{array}$ & $\begin{array}{c}41.34 \\
(100 \%)\end{array}$ & $\begin{array}{c}45.66 \\
(110 \%)\end{array}$ \\
\hline & Mem. & $\begin{array}{c}35.27 \\
(100 \%)\end{array}$ & $\begin{array}{c}8.35 \\
(24 \%)\end{array}$ & $\begin{array}{c}35.27 \\
(100 \%)\end{array}$ & $\begin{array}{c}8.35 \\
(24 \%)\end{array}$ \\
\hline & Total & $\begin{array}{c}72.23 \\
(100 \%)\end{array}$ & $\begin{array}{l}48.86 \\
(68 \%)\end{array}$ & $\begin{array}{c}76.61 \\
(100 \%)\end{array}$ & $\begin{array}{l}54.01 \\
(71 \%)\end{array}$ \\
\hline
\end{tabular}

* Forward memory is compiled memory in Samsung $65 \mathrm{~nm}$ CMOS process

* All ACS units were operated at fixed frequency of $400 \mathrm{MHz}$

$\dagger$ The number of stored metrics of sixteen ACS units at one state

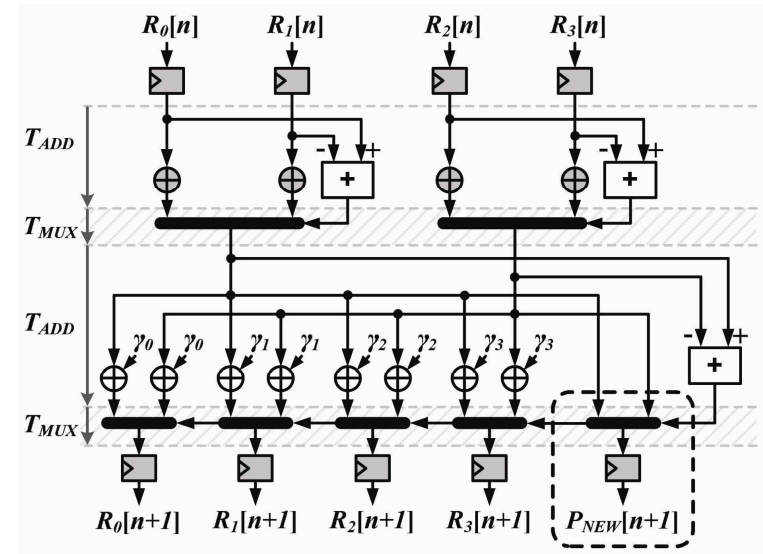

Fig. 11. Architecture of Type-C3: modified Type-C2 with adding the recovery circuit which is shown in dotted line.

circuit in Type-C3 and Type-L3, Eqs. (15) and (16), is added to Eqs. (6) and (13), respectively.

$$
\begin{gathered}
P_{N E W . C 3}[n+1]=\max \left\{R_{\alpha}, R_{\beta}\right\} \\
P_{N E W . L 3}[n+1] \\
=\max \left(R_{0}[n]-P_{N O R M}, R_{1}[n]-P_{N O R M},\right. \\
\left.R_{2}[n]-P_{N O R M}, R_{3}[n]-P_{N O R M}\right)
\end{gathered}
$$

Even though the cost of hardware overhead incurred by recovery circuit is increased in terms of one MUX and one register, the critical path of Type-C3 and Type-L3 is maintained to have same delay with that of Type-C2 and Type-L2, respectively. The requirement of forward memory which stores original path metric instead of recursive metrics is hugely reduced by more than $75 \%$ compared to previous retimed radix-4 SISO decoder architectures without recovery circuit. In addition, since all path metrics are normalized by subtracting the $P_{\text {NORM }}$ which is same with one of the eight path metrics at the same state for avoiding overflow, one path metric among the eight path metrics does not need to be stored. The number of metrics to be stored can be reduced from 8 to $7[16]$.

\section{EXPERIMENTAL RESULTS}

In this Section IV, experimental results are presented such as minimum achievable delay, area, and power consumption for the cascaded radix-4 ACS units and the LUT-based radix-4 ACS units in the post-layout implementation. The minimum achievable delay means the maximum operating clock frequency. The six types of radix-4 ACS units, Type- $\{C 1, C 2, C 3, L 1, L 2, L 3\}$, were described in Verilog HDL and implemented in Samsung $65 \mathrm{~nm}$ CMOS process.

\section{Comparison among Retimed Results}

Reducing the critical path delay of radix-4 ACS units leads to the increase of hardware complexity. The common operator migration and retiming technique leads to hardware complexity increment as the amount of increased hardware: 4 times of normalization adder, 2 times of branch metric adder for the Type-C2 and 4 times for the Type-L2, and several MUXs.

In Fig. 12, the gate count is indicated with minimum achievable delay for Type-\{Cl,C2\} and Type- $\{L 1, L 2\}$. This figure represents the changes of gate count and minimum achievable delay caused by applying retiming technique. Accordingly, the minimum achievable delay of Type-C1 can be reduced by $34 \%$ and $42 \%$ at the expense of $52 \%$ and $122 \%$ hardware overhead for Type$C 2$ and Type-L2, respectively. The amount of increased hardware overhead in Type-L2 is huge compared to that 


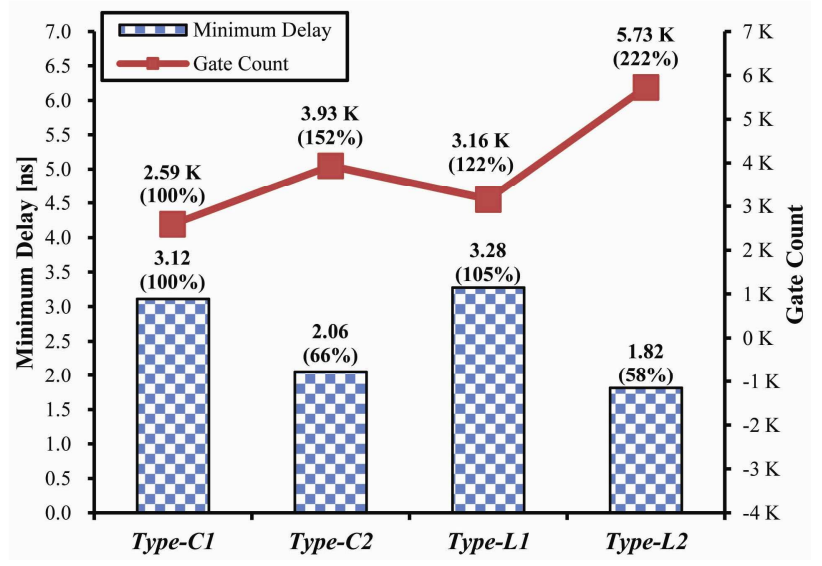

Fig. 12. The NAND2 equivalent gate count and minimum achievable delay comparison between one retimed radix-4 ACS unit (Type-\{C2,L2\}) and one original radix-4 ACS unit (Type$\{C 1, L 1\})$.

of Type-C2, while the amount of reduced minimum delay is approximately equal.

By the result, the retimed cascaded radix-4 ACS unit, Type-C2, is the best choice for high-throughput radix-4 SISO decoding operation to minimize the hardware overhead and power consumption.

\section{Implementation Results}

In Table 3, the implementation results in terms of forward memory requirement, area, and power consumption are compared between the proposed architecture (Type- $\{C 3, L 3\}$ ) and the non-recovery circuit architecture (Type-\{C2,L2\}) at the operating frequency of $400 \mathrm{MHz}$. With the proposed path metric recovery technique, the forward memory size is reduced by $78 \%$ for both cascaded type and LUT-based type at the expense of $6 \%$ and $4 \%$ hardware overhead of Type-C3 and Type-L3, respectively, and $10 \%$ power consumption increment of both Type-C3 and Type-L3. We obtained the huge reduction of forward memory requirement from the cost of small increase of hardware resources and power consumption. The total area is reduced by $47 \%$ and $43 \%$ with the reduction of $32 \%$ and $29 \%$ power consumption for the path metric calculation logic with forward memory based on Type-C3 and Type-L3, respectively.

Therefore, the proposed SISO decoding architecture can reduce efficiently the forward memory requirement, hardware complexity, and power consumption. In detail, since the area and power consumption of cascaded ACS architecture is smaller than that of LUT-based ACS architecture with similar critical path delay, the proposed Type-C3 is appropriate to be exploited for highthroughput radix-4 SISO decoder with low memory requirement.

\section{CONCLUSIONS}

To obtain the high-throughput radix-4 SISO decoding architecture with reduced memory requirement, path metric recovery technique is proposed. This technique is applied on the retimed radix-4 ACS units, and analyzed for finding the most appropriate high-throughput radix-4 SISO decoding architecture. The proposed recovery circuit eliminates the need to store four metrics of one retimed radix-4 ACS unit to the forward memory. It recovers the original path metric and reduces the entire forward memory size by $78 \%$ with small increase of hardware overhead. The comparison results between cascaded radix-4 ACS architecture and LUT-based radix4 ACS architecture is presented in terms of delay, area, and power consumption. Even though the retimed cascaded radix-4 ACS unit and the retimed LUT-based radix-4 ACS unit have similar reduced critical path delay, the increase of hardware overhead and power consumption is represented to have significant difference. The retimed cascaded radix-4 ACS unit is effective in reducing the total hardware complexity and power consumption based on recovery circuit compared to the retimed LUT-based radix-4 ACS unit. The proposed architecture which is applied on the cascaded radix-4 ACS and LUT-based radix-4 ACS is implemented in Samsung $65 \mathrm{~nm}$ CMOS process.

\section{ACKNOWLEDGMENTS}

This research was supported by IDEC, Basic Science Research Program through the National Research Foundation of Korea(NRF) funded by the Ministry of Science, ICT \& Future Planning(2012R1A1A1010064), and by the Center for Integrated Smart Sensors funded by the Ministry of Science, ICT \& Future Planning as Global Frontier Project (CISS- 2011-0031860). 


\section{REFERENCES}

[1] Z. Wang, Z. Chi, and K. Parhi, "Area-efficient high-speed decoding schemes for turbo decoders," IEEE Trans. Very Large Scale Integr. (VLSI) Syst., vol. 10, no. 6, pp. 902-912, Dec. 2002.

[2] J. Hsu and C. Wang, "A parallel decoding scheme for turbo codes," in Proc. IEEE Int. Conf. Circuits and Systems, vol. 4, 1998, pp. 445-448.

[3] B. Bougard, A. Ciulietti, L. V. d. Perre, and F. Catthoor, "A class of power efficient VLSI architectures for high speed turbo-decoding," in Proc. IEEE Global Telecommunication Conf., vol. 1, 2002, pp. 553-549.

[4] B. Bougard et al., "A scalable $8.7 \mathrm{~nJ} / \mathrm{bit} 75.6 \mathrm{Mb} / \mathrm{s}$ parallel concatenated convolutional (turbo-) codec," in IEEE Int. Solid-State Circuits Conf. (ISSCC) Dig. Tech. Papers, 2003, pp. 152-153.

[5] A. Worm, H. Lamm, and N. Wehn, "A high-speed MAP architecture with optimized memory size and power consumption," in Proc. IEEE Workshop on Signal Processing Systems (SiPS2000), 2000, pp. 265-274.

[6] A. Worm, H. Lamm, and N. Wehn, "Design of low-power high-speed maximum a priori decoder architectures," in Proc. Design, Automation and Test in Eur. Conf. Exhibition, Apr. 2001, pp. 258265.

[7] M. M. Mansour and N. R. Shanbhag, "Design methodology for highspeed iterative decoder architectures," in Proc. IEEE Int. Conf. Acoustics, Speech, and Signal Processing, vol. 3, 2002, pp. 3085-3088.

[8] M. M. Mansour and N. R. Shanbhag, "VLSI architectures for SISO-APP decoders," IEEE Trans. Very Large Scale Integr. (VLSI) Syst., vol. 11, no. 4, pp. 627-650, Aug. 2003.

[9] A. Giulietti et al., "Parallel turbo code interleavers: Avoiding collisions in accesses to storage elements," Electron. Lett., vol. 38, no. 5, pp. 232234, Feb. 2002.

[10] S.-J. Lee, N. Shanbhag, and A. Singer, "Areaefficient high-throughput map decoder architectures," IEEE Trans. Very Large Scale Integr. (VLSI) Syst., vol. 13, no. 8, pp. 921-933, Aug. 2005.

[11] Z. Wang, "High-speed recursion architectures for
MAP-based turbo decoders," Very Large Scale Integration (VLSI) Systems, IEEE Transactions on, vol. 15, no. 4, pp. $470-474$, april 2007.

[12] C.-H. Tang, C.-C. Wong, C.-L. Chen, C.-C. Lin, and H.-C. Chang, "A 952ms/s Max-Log MAP decoder chip using radix-4 x 4 ACS architecture," in Solid-State Circuits Conf., 2006. ASSCC 2006. IEEE Asian, nov. 2006, pp. $79-82$.

[13] K.-T. Shr, Y.-C. Chang, C.-Y. Lin, and Y.-H. Huang, "A 6.6pj/bit/iter radix-16 modified logMAP decoder using two-stage ACS architecture," in Solid State Circuits Conf. (A-SSCC), 2011 IEEE Asian, nov. 2011, pp. 313 -316.

[14] O. Sanchez, C. Jegoy, M. Jezequel, and Y. Saouter, "High speed low complexity radix-16 Max-LogMAP SISO decoder," Electronics, Circuits and Systems, 2012, ICECS 2012, 19th IEEE Int. Conf. on, 9-12, pp.400-403, Dec., 2012.

[15] K. Parhi, VLSI Digital Signal Processing Systems: Design and Implementation. New York: Wiley, 1999.

[16] J. H. Kim, "Memory reduced MAP decoding for double-binary turbo decoder," IEICE Trans. Electron., vol. 8, no. 23, pp. 1996-2001, Dec. 2011.

[17] S. J. Lee, "High speed add-compare-select circuit for radix-4 Viterbi decoder," U.S. Patent 2004/0122883(A1), June 24, 2004.

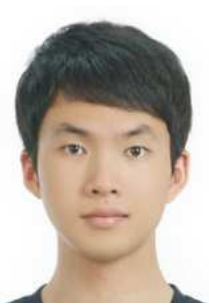

Wooseok Byun received the B.S. degree in the Department of Electronics Engineering in 2013, from Chungnam National University, Daejeon, Korea, where he is currently working toward the M.S degree. His interests include lowpower digital VLSI circuit and SoC design.

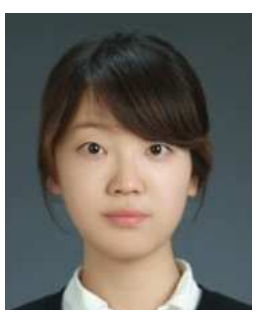

Hyeji Kim received the B.S degree in the Department of Electronics Engineering in 2013, from Chungnam National University, Daejeon, Korea, where he is currently working toward the M.S degree. Her current research interests include the design of VLSI architectures for communication modem and digital signal processing. 
Ji-Hoon Kim received the B.S. (summa cum laude) and Ph.D. degrees in electrical engineering and computer science from KAIST, Daejeon, Korea, in 2004 and 2009, respectively. His Ph.D. work focused on design of high performance CPU and baseband modem for mobile hand-held devices. In 2009, he joined Samsung Electronics, Suwon, Korea, where he worked on the SoC architecture design for next generation cellular modem. He is currently with Chungnam National University, Daejeon, Korea, as an Assistant Professor. $\mathrm{He}$ is a member of the steering committee of the IC Design Education Center (IDEC), KAIST. His current interests include embedded processor, communication modem, and ultra low power SoC design for IoT (Internet of Things) devices. 